\title{
Developing fiber lasers with Bragg reflectors as deep sea hydrophones
}

\author{
Nicolò Beverini $\left({ }^{1}\right)\left({ }^{3}\right)$, Riccardo Falciai $\left({ }^{2}\right)$, Enrico Maccioni $\left({ }^{1}\right)\left({ }^{3}\right)$, Mauro Morganti $\left({ }^{1}\right)\left({ }^{3}\right)$, \\ Fiodor Sorrentino $\left({ }^{1}\right)$ and Cosimo Trono $\left({ }^{1}\right)\left({ }^{2}\right)$ \\ (1) Dipartimento di Fisica «E. Fermi», Università degli Studi di Pisa, Italy \\ ${ }^{2}{ }^{2}$ Istituto di Fisica Applicata «Nello Carrara» (IFAC), CNR, Firenze, Italy \\ $\left(^{3}\right)$ Istituto Nazionale di Fisica Nucleare (INFN), Sezione di Pisa, Italy
}

\begin{abstract}
The present paper will discuss the work in progress at the Department of Physics of the University of Pisa in collaboration with the IFAC laboratory of CNR in Florence to develop pressure sensors with outstanding sensitivity in the acoustic and ultrasonic ranges. These devices are based on optically-pumped fiber lasers, where the mirrors are Bragg gratings written into the fiber core.
\end{abstract}

Key words hydrophones - Bragg fiber-laser - pressure sensors

\section{Introduction}

In the field of acoustic detection, piezoelectric sensors are the most widely used devices. This well established technology offers high reliability and relatively low cost production. Sensors based on optical fibers have well known advantages over conventional electro-mechanical sensors. They offer electrically passive operation and immunity from electromagnetic fields, since the fiber is realized entirely with dielectric materials (glass and plastic). They have very small dimensions (outer diameter $\sim 125 \mu \mathrm{m}$ for a standard fiber), and have multiplexing capabilities for a quasi-distributed measurement configuration, by using a single opto-electronic control unit. Remote measurement is also possible. Indeed, the very low signal attenuation $(\sim 0.3 \mathrm{~dB} / \mathrm{km})$ of the

Mailing address: Dr. Nicolò Beverini, Dipartimento di Fisica «E. Fermi», Università degli Studi di Pisa, Largo Pontecorvo 3, 56127 Pisa, Italy; e-mail: beverini@df.unipi.it fibers in the region around $1.55 \mu \mathrm{m}$ makes it possible to place the opto-electronic control unit several $\mathrm{km}$ far from the measurement point. Moreover, high sensitivity and wide dynamic measurement range can usually be achieved.

\section{Fiber Bragg Grating sensors}

A Fiber Bragg Grating (FBG) consists of a periodic perturbation of the optical fiber core refractive index. This grating acts on the radiation traveling in the fiber as a wavelength selective mirror. If the grating pitch is $\Lambda$, the reflection band is peaked at a wavelength

$$
\lambda_{\text {Bragg }}=2 n_{\text {eff }} \Lambda
$$

where $\lambda_{\text {Bragg }}$ is the so-called Bragg wavelength and $n_{\text {eff }}$ is the effective refractive index. The width of the reflection band can be regulated by controlling the reflectivity of the mirror, that is by controlling the length of the grating (its total number of lines) and the depth of the reflection index modulation. Typical reflection bandwidth values are around $0.2 \mathrm{~nm}$. Radiation outside the Bragg resonance condition will propagate in the fiber without perturbations (fig. 1). 


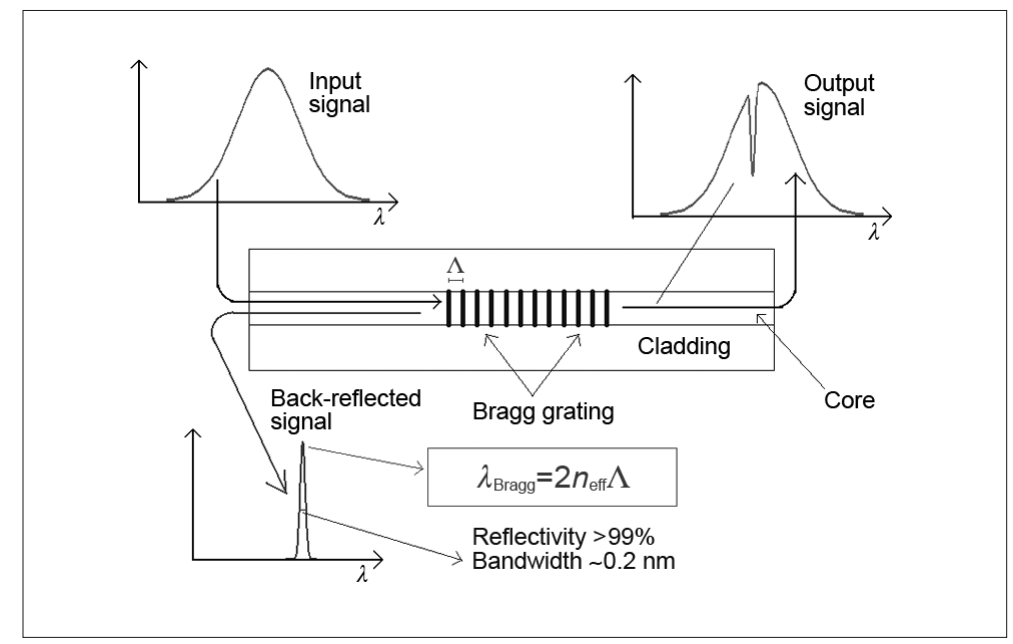

Fig. 1. Fiber Bragg grating reflection and transmission properties.

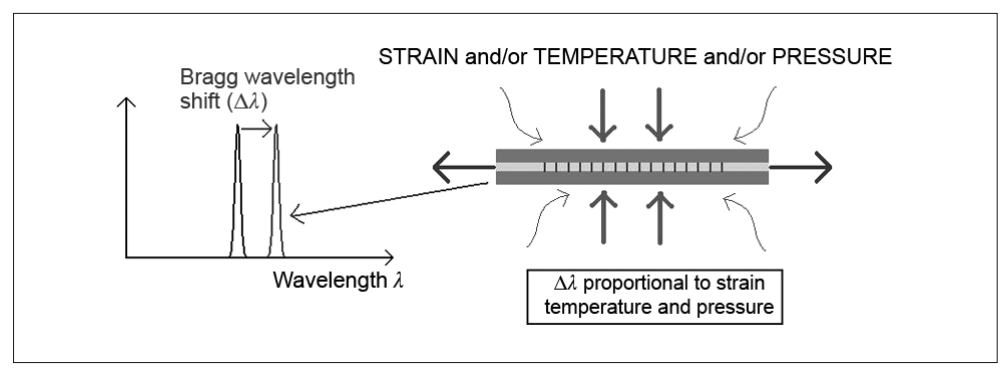

Fig. 2. Strain, temperature and pressure effects on a Bragg grating reflection spectrum.

FBGs are fabricated using the phase-mask technique on special Ge-doped fibers (Hill and Meltz, 1997). A phase-mask is a diffractive optical element which spatially modulates the UV writing beam (typically at $\lambda=248 \mathrm{~nm}$, where the photosensitivity of the fiber is at its best). The near-field fringe pattern, which is produced behind the mask, photo-imprints a refractive index modulation on the core of the photosensitive fiber.

These gratings are highly sensitive to external perturbations that affect the fiber. With regard to eq. (2.1), every strain and temperature or pressure variation on the fiber changes both the grating pitch and the refractive index, producing a shift in the Bragg wavelength (fig. 2). The information is encoded on the wavelength, with the considerable advantage that a number of different gratings, with different Bragg wavelengths, can be inscribed on the same fiber and interrogated by the same opto-electronic unit, thus enabling a quasi-distributed measurement. The typical sensitivities of a fiber Bragg grating to strain, temperature, and pressure (which acts as an isotropic strain) are

strain: $\frac{\Delta \lambda_{\text {Bragg }}}{\lambda_{\text {Bragg }}}=\left\{1-\frac{n_{\text {eff }}^{2}}{2}\left[p_{12}-v\left(p_{11}-p_{12}\right)\right]\right\} \varepsilon$ 
temperature: $\frac{\Delta \lambda_{\text {Bragg }}}{\lambda_{\text {Bragg }}}=\left(\alpha_{\Lambda}+\alpha_{v}\right) \Delta T$

pressure: $\frac{\Delta \lambda_{\text {Bragg }}}{\lambda_{\text {Bragg }}}=\left[-\frac{(1-2 v)}{E}+\frac{n^{2}}{2 E}(1-2 v)\right.$.

$$
\left.\cdot\left(2 p_{12}+p_{11}\right)\right] \Delta P
$$

where $n_{\text {eff }}$ is the fiber effective refractive index, $v$ is Poisson's ratio, $\varepsilon=\Delta L / L$ is the strain (Bragg grating length $L), p_{i j}$ are Pockel's coefficients of the stress-optic tensor, $\alpha_{\Lambda}$ is the thermal expansion coefficient, $\alpha_{n}$ represents the thermo-optic coefficient, and $E$ is Young's modulus. For silica fibers, at $1550 \mathrm{~nm}$ the strain sensitivity is $\sim 1.2$ $\mathrm{pm} / \mu \varepsilon$, the temperature sensitivity is $\sim 10 \mathrm{pm} /{ }^{\circ} \mathrm{C}$, and the pressure sensitivity is $\sim-3.6 \mathrm{pm} / \mathrm{MPa}$.

A typical FBG sensor device includes a broad-spectrum source (i.e. a superluminescent LED with $\sim 40 \mathrm{~nm}$ spectral emission width). Its radiation is coupled into the fiber, and interacts with the grating. The wavelength shift of the reflection peak is then detected by means of spectro-photometric methods.

FBGs sensors are robust, can have a wide dynamic range, and can be easily multiplexed in order to realize multipoint detection at a low cost. Their ultimate sensitivity is limited, however, by the spectral bandwidth of the Bragg grating which at best can be of the order of $0.2 \mathrm{~nm}$.

A typical application for FBG sensors is the control of deformations in structures to which the gratings can be attached or within which they can be embedded. The following is a summary of FBG sensor applications made by the IFAC group:

- Applications for the cultural heritage (Castelli et al., 2003): in situ measurement and continuous monitoring of deformations in painted wood panels.

- Automotive applications: monitoring of deformations in car windshields (Falciai et al., 2004).

- Structural health monitoring: FBGs embedded in carbon fibers in composite strips for concrete beam reinforcement and monitoring (Falciai et al., 2005).

FBGs show only a limited responsivity to variations in pressure (the typical wavelength resolution of FBG interrogation systems is $\sim 1$ pm, which means approximately $0.3 \mathrm{MPa}$, or $3 \mathrm{~atm})$. Therefore, their typical application is for high pressure sensing (Xu et al.,1993).

\section{Fiber lasers}

A Distributed Bragg Reflector Fiber Laser (DBR-FL) consists of two Bragg gratings with identical reflection wavelengths (Ball and Glenn, 1992), which are directly inscribed in a singlemode erbium-doped optical fiber (fig. 3). When pumped with $980 \mathrm{~nm}$ radiation, this structure acts as an active medium inserted in a Fabry-Perot laser cavity, with an emission peak around 1530 $\mathrm{nm}$. The power emitted is a function of the broadband source pumping power, the length of the laser cavity and the Bragg gratings reflectivity,

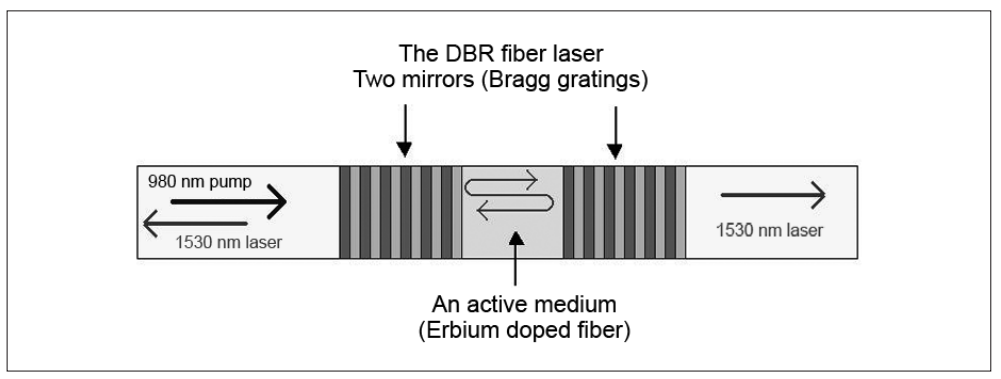

Fig. 3. Fiber laser pumping scheme. 
but it is in any case some orders of magnitude higher than the power available on the reflected radiation from a passive FBG sensors. Cavity length is an important laser parameter. The emitted laser power increases with the cavity length, but a shorter length means a larger separation between the resonant longitudinal modes $(\lambda=$ $=\lambda^{2} / 2 L$ ). With a cavity length of a few $\mathrm{cm}$, only one cavity mode lies inside the optical bandwidth of the Bragg gratings, and it becomes possible to operate with the laser in a stable single-frequency emission mode. In these conditions the laser linewidth is very narrow ( $<5 \mathrm{kHz}$, equivalent to a coherence length of over $30 \mathrm{~km}$ ). The comparison between the performances of devices using the FBG and the DBR is provided in table I.

The very narrow line-width means that the DBR laser sensor is intrinsically more sensitive than the FBG sensor; for this reason, it seems particularly suitable for very low-pressure measurements.

Table I. Comparison of FBG and DBR performances.

\begin{tabular}{lccc}
\hline \hline & Power & $\begin{array}{c}\text { Line-width } \\
(\mathrm{kHz})\end{array}$ & $\begin{array}{c}\text { Line-width } \\
(\mathrm{nm})\end{array}$ \\
\hline FBG (passive) & $1-100 \mathrm{nW}$ & $\sim 10^{7}$ & $\sim 0.1$ \\
DBR (active) & $100 \mu \mathrm{W}-1 \mathrm{~mW}$ & $\sim 5$ & $\sim 5 \cdot 10^{-8}$ \\
\hline
\end{tabular}

DBR fiber lasers were fabricated in the IFAC laboratories. An excimer (KrF) UV laser, with emission at $248 \mathrm{~nm}$, was used for Bragg gratings writing. We obtained a Bragg grating with $\lambda_{\text {Bragg }} \cong 1532 \mathrm{~nm}$ by a phase mask with a nominal spatial period of $1059.9 \mathrm{~nm}$ on an erbium doped fiber having an absorption coefficient of $14 \mathrm{~dB} / \mathrm{m}$ at $980 \mathrm{~nm}$. The length of Bragg gratings was $1 \mathrm{~cm}$, and we chose the distance between the two FBG reflectors and the FBGs reflectivity in order to obtain the maximum power emission in stable, single longitudinal mode regime. Typical values are: output FBG reflectivity: >90\%; back FBG reflectivity: >99\%; distance between the FBGs: $2 \mathrm{~mm}-2 \mathrm{~cm}$; emission power: $10 \mu \mathrm{W}-1 \mathrm{~mW}$ with about $200 \mathrm{~mW}$ of $980 \mathrm{~nm}$ pumping power.

The laser line-width was measured by heterodyning on a fast photodiode the beams from two different lasers with slightly different emission wavelengths, and then observing the resulting beat note on a radio-frequency spectrum analyzer. We could estimate a line-width narrower than $5 \mathrm{kHz}$.

It is possible to inscribe several laser structures on a single doped fiber with the aim of multiplexing a few sensors, by working at slightly different wavelengths on the same fiber with a common pump laser. In order to test multiplexing operation, we wrote two lasers on the same optical fiber with a different grating pitch. Figure 4

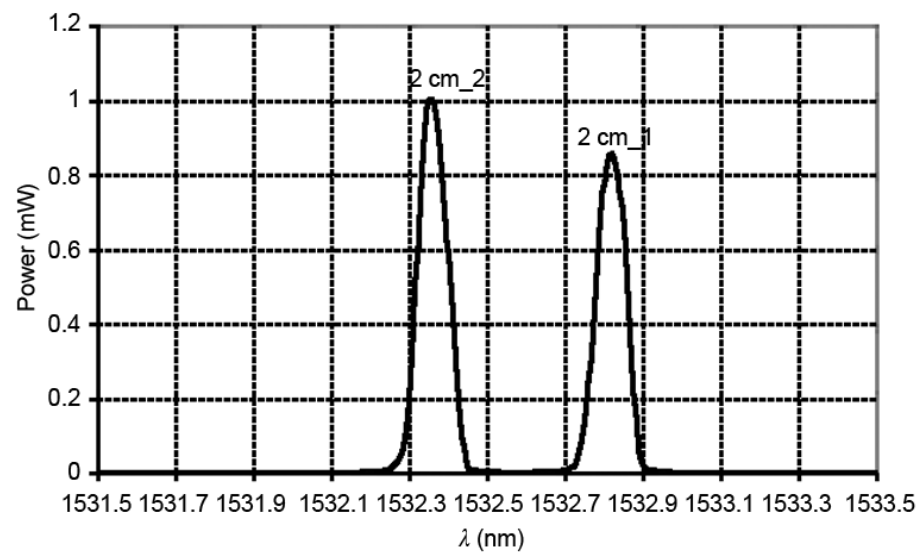

Fig. 4. Emission spectrum of an array of two fiber lasers. 


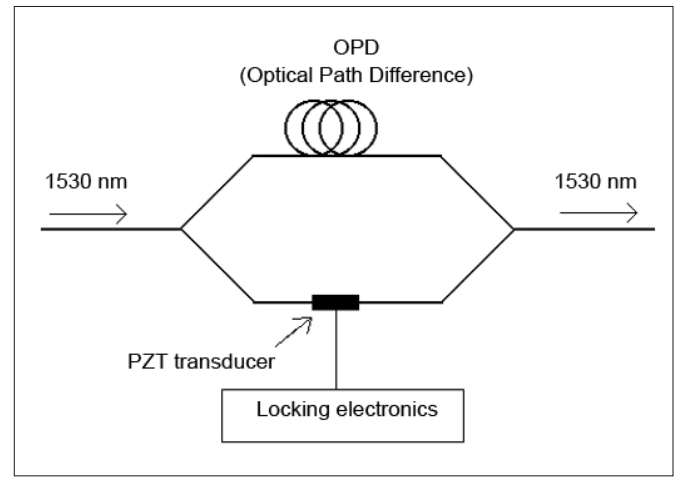

Fig. 5. The Mach-Zender Interferometer.

reports the emission spectrum of this two-laser array, acquired by means of an optical spectrum analyzer (wavelength resolution: $0.1 \mathrm{~nm}$ ).

\section{Fiber laser hydrophone}

In developing highly sensitive hydrophones for deep sea applications, the ultimate goal is to achieve a sensitivity at the level of the acoustic background noise of the quiet ocean, which is conventionally represented by the so-called Deep Sea State Zero (DSS0) (Wenz, 1962). At $1 \mathrm{kHz}$, the DSSO level is $100 \mu \mathrm{Pa} / \mathrm{Hz}^{1 / 2}$, which should correspond (eq. (2.4)) to a wavelength shift of approximately $10^{-12} \mathrm{~nm}, 11$ orders of magnitude less than the typical FBG bandwidth. The DBR fiber laser emits monochromatic radiation with a line-width of approximately $4 \cdot 10^{-8} \mathrm{~nm}$, and an interferometric detection technique provides the possibility of pushing the sensitivity a factor $10^{3}-10^{4}$ below the laser line-width, thus approaching the sensitivity required by the DSS0 level. Figure 5 shows the scheme of an unbalanced Mach-Zender interferometer (MZI), which we used in our detection scheme.

The interferometer converts the pressure-induced wavelength shift of the radiation emitted by the DBR fiber laser into a phase delay. The phase difference $\Delta \varphi_{\mathrm{MZ}}$ at the MZI output is dependent on the fiber laser output wavelength shift
$\Delta \lambda$ and on the Optical Path Difference (OPD= $=n L$ ), which is the length difference $L$ of the two interferometer arms times the fiber core refractive index $n$. The following relationship holds:

$$
\Delta \varphi_{\mathrm{MZ}}=\frac{2 \pi \cdot \mathrm{OPD}}{\lambda^{2}} \Delta \lambda
$$

With $\lambda$ of the order of $10^{-12} \mathrm{~nm}$ (DSS0 conditions), an OPD of $300 \mathrm{~m}$ gives a value of $\varphi_{\mathrm{MZ}} \approx 1 \mu \mathrm{rad}$, which is a resolution achievable with the present technology (Kersey et al., 1992).

\section{Experimental tests}

We made a preliminary characterization of the sensor in our laboratory and tested the responsivity of our lasers by using a MZI with OPDs of $150 \mathrm{~m}$ and $450 \mathrm{~m}$. According to eq. (4.1), the MZI responsivity increases by increasing the unbalance, up to the limit given by the coherence length of the laser radiation, which for these lasers consists of many $\mathrm{km}$. The monotonic operative range and the dynamic range are, however, reduced proportionally. Indeed, the phase detector signal is not univocally related to the wavelength shift, when the phase difference grows over $2 \pi$ radians, and the low frequency noise of the laser exceeds this limit, yet with a few meter of length unbalance. We were then obliged to lock the MZI phase to the laser frequency at low Fourier frequencies, with a cut-off of the order of several $\mathrm{kHz}$, by using a servo loop that acts on the length of one arm of the interferometer by stretching the fiber through a piezoelectric actuator. The interferometer was used in the condition usually defined as «quadrature» detection (Kersey et al., 1992), with the MZI locked at one side of a fringe in the middle point, where the sinusoidal function is proportional to the phase and the responsivity has its maximum value.

The experimental apparatus is sketched in fig. 6. The fiber laser was sink in a $1 \mathrm{~m}^{3}$ water tank, close to a conventional Piezo-Transducer (PZT) hydrophone for calibration and validation; a SDR HS/150 model was used with a nominal responsivity of $1.778 \mathrm{mV} / \mathrm{Pa}(29 \mathrm{~dB}$ 


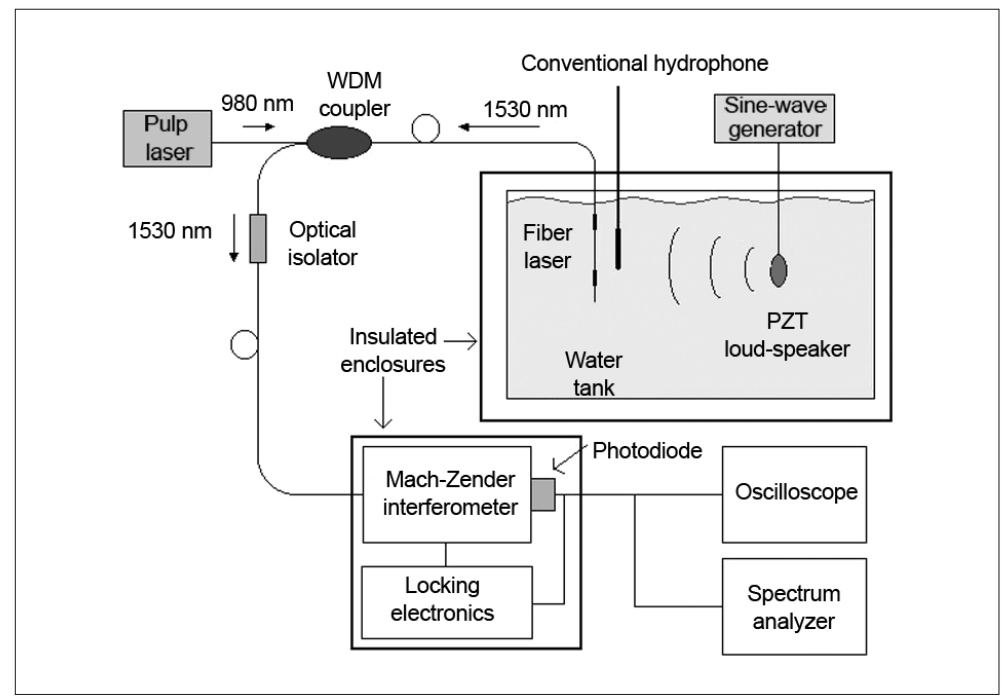

Fig. 6. Experimental setup.

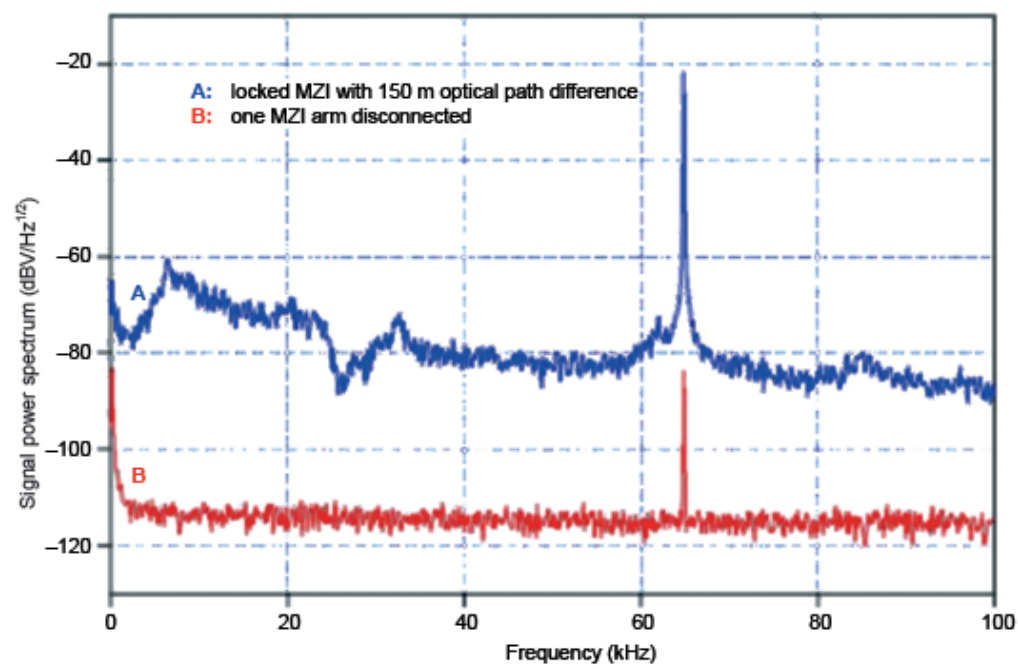

Fig. 7. Fiber laser spectral response to a $65 \mathrm{KHz}$ test tone $(\mathrm{OPD}=150 \mathrm{~m})$. Top $(\mathrm{A})$ : the fiber laser response (output of the MZI) to a $65 \mathrm{kHz}$ loud-speaker acoustic test note. Bottom (B): the laser output spectrum (without the MZI).

amplification). A PZT loud-speaker provided a cw sinusoidal uncalibrated acoustic signal. The laser emission wavelength modulation, induced by the acoustic signal, was converted into phase modulation by the Mach-Zender interferometer, and analyzed by means of an electronic spectrum analyzer $(125 \mathrm{~Hz}$ resolution bandwidth). 
Figure 7 compares the MZI output with the laser output spectrum in the absence of the interferometer when the fiber laser was stimulated with a $65 \mathrm{kHz}$ acoustic wave. From these two spectra we deduced a limit for the performance of a particular FL sensor, referred to a device of a specific optical power $(100 \mu \mathrm{W})$, and a fixed orientation with respect to the acoustic source. In fact the upper base line $-80 \mathrm{dBV} / \mathrm{Hz}^{1 / 2}$ level, corresponding to about $56 \mathrm{mPa} / \mathrm{Hz}^{1 / 2}$, was produced by the actual environmental noise level. The bottom $-118 \mathrm{dBV} / \mathrm{Hz}^{1 / 2}$ level, which corresponds to about $0.7 \mathrm{mPa} / \mathrm{Hz}^{1 / 2}$, represented the opto-electronic noise level, and thus the lowest

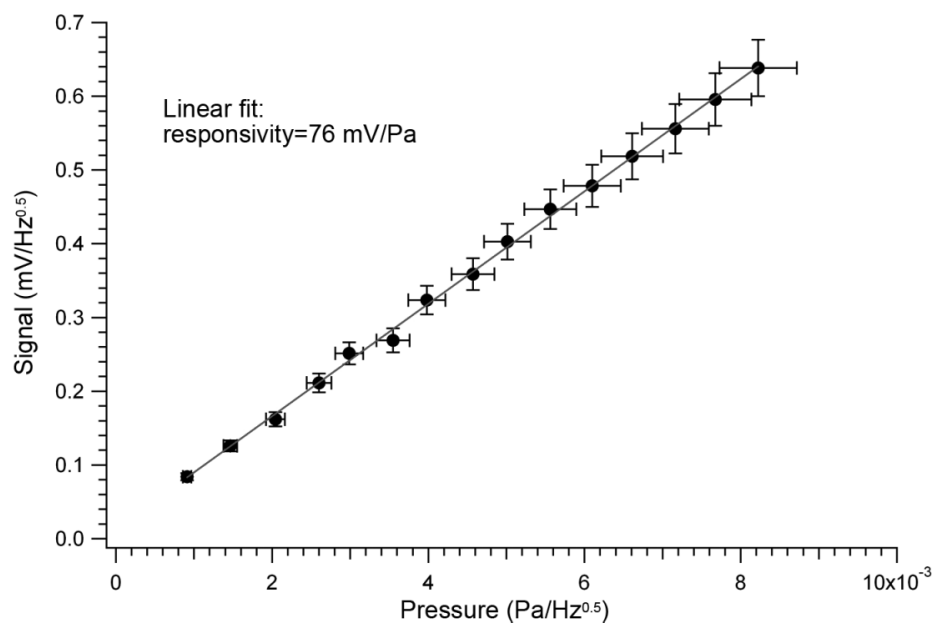

Fig. 8. FL output signal versus pressure (measured with the calibrated reference hydrophone). FL optical power $10 \mu \mathrm{W}$, test note frequency $40 \mathrm{kHz}$; OPD $\sim 450 \mathrm{~m}$. Error bars represent the reading uncertainty.

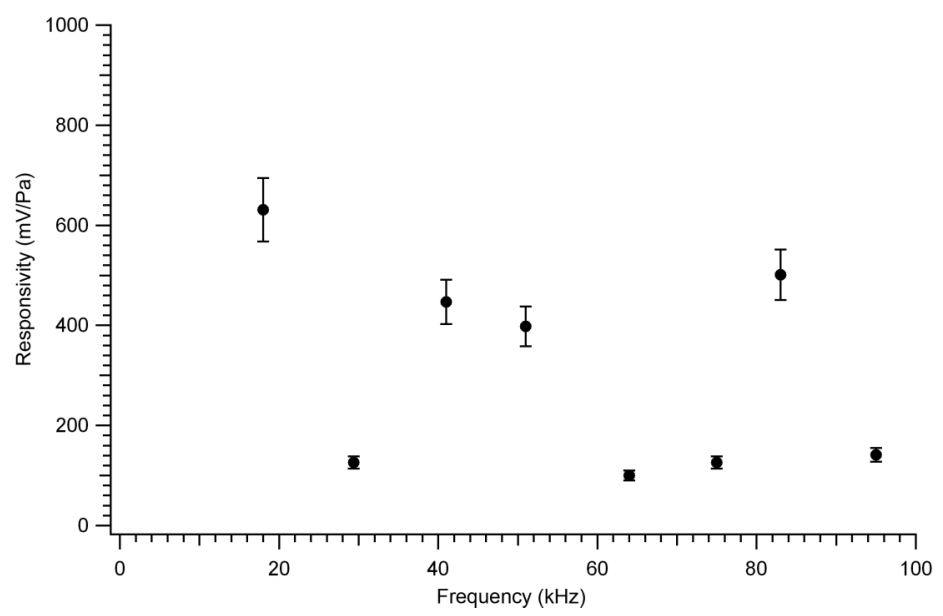

Fig. 9. FL responsivity for different test note frequencies; OPD $\sim 450 \mathrm{~m}$; FL optical power $10 \mu \mathrm{W}$. 
attainable limit for the minimum detectable signal of the fiber laser. The acoustic level in $\mathrm{mPa}$ was estimated by means of a comparison with the readings of a calibrated PZT hydrophone.

We measured the response of the device to in-water acoustic notes at several fixed frequencies in the region between about 15 and 100 $\mathrm{kHz}$, for different intensities of loud-speaker excitation. By making a comparison with the calibrated PZT hydrophone, we observed a highly linear trend of the FL output signal versus pressure (fig. 8). In this particular experimental condition, we measured a responsivity of $76 \mathrm{mV} / \mathrm{Pa}$ at $40 \mathrm{kHz}$, where the voltage value refers to the photodiode signal at the MZI output. The reading error bars $( \pm 0.5 \mathrm{db})$ on the single measurement are reported.

However, the responsivity is changing significantly as a function of the acoustic wave frequency. Figure 9 reports measured responsivities for a same FL and a same Mach-Zender OPD. The large spread for the responsivity values depends strongly on the particular set of experimental parameters. In fact, it can be affected at some specific frequencies by the acoustic reflections between the walls of the water tank, due to its reduced dimensions. Also mechanical resonances of the fiber laser holder, and of the fiber itself, may have caused a dependence of the frequency response. Moreover, the acoustic perturbation in water may act differently on the two Bragg gratings of the laser, because they are spatially separated. All these factors dictate that a true characterization of the sensors should be made only under better control of experimental conditions, operating in a large pool and in pulsed excitation regime, in order to avoid reflection effects. In particular, it will be important to study the dependence of the response on the orientation of the sensor. In any case, we can say that the pressure dynamic range for the FL sensor set-up shown in figs. 8 and 9 is roughly of four decades. In fact, the minimum detectable pressure in this experimental condition is of the order of $1 \mathrm{mPa} / \mathrm{Hz}^{1 / 2}$ at every frequency; this is, for example, the measured value of the first point on fig. 8. The output voltage of the device is linear with pressure up to about $1 \mathrm{~V}$, that is, up to $10 \mathrm{~Pa}$ with minimum responsivity in the $15-100 \mathrm{kHz}$ range of approximately $100 \mathrm{mV} / \mathrm{Pa}$ (fig. 9).

\section{Conclusions}

The results of the study, development and experimental validation of a DBR fiber laser for acoustic sensing in marine environment, have been reported. Single mode lasers were fabricated by writing two Bragg gratings on an erbium-doped fiber core. The very narrow linewidth $(<5 \mathrm{kHz})$, combined with an interferometric detection, could make possible a wavelength resolution of $\sim 10^{-12} \mathrm{~nm}$. The comparison with a calibrated PZT hydrophone $(15-100 \mathrm{kHz}$ frequency range) showed a highly linear trend of the fiber laser output signal versus pressure. On the other hand, FL responsivity was strongly frequency dependent. This fact was probably connected to the particular experimental set-up, such as the acoustic reflections between the walls of the water tank and the mechanical resonance of the FL holder and of the fiber itself.

The FBG hydrophones offer a wide range of applications, ranging from the marine environmental acoustic monitoring to the deep-sea study and the survey of dolphins and whales. In general, a network of multiplexed FBG hydrophones could provide much information on what happens in the «under-the-sea world» in the acoustic and ultrasound regimes, whichever could be the origin of the propagating pressure wave. An interesting field of application could be the acoustic detection of particle showers produced in water by very high-energy neutrinos, as an alternative method to the more conventional photo-detection.

\section{Acknowledgements}

This research was supported by the Italian University Ministry (MIUR), by the University of Pisa, by National Institute of Nuclear Physics (INFN), and by the Italian National Research Council (CNR).

\section{REFERENCES}

BaLl, G.A. and W.H. GlenN (1992): Design of a singlemode linear-cavity erbium fiber laser utilizing Bragg reflectors, J. Lightwave Tech., 10, 1338-1343.

Castelli, C., G. Lanterna, R. Falciai and C. Trono 
(2003): Continuous monitoring of wooden works of art using fiber Bragg-grating sensors, J. Cultural Heritage, 4, 285-290.

Falciai, R., C. Trono, P. Castelli, R. Galli, N. Mattiucci and N. PALlaro (2004): Automotive applications of fiber Bragg grating sensors, in Proceedings of the 8th Conference Sensors and Microsystems «Aisem 2003», edited by C. Di Natale, A. D’Amico, G. Soncini, L. Ferrario and M. ZEN (World Scientific Publisher), p. 545.

Falciai, R., J.M. Kenny, A. Terenzi, C. Trono and R. MEZZACASA (2005): Reinforcing and monitoring of concrete structures with composites and fiber optic sensors, in Proceedings of the 9th Conference Sensors and Microsystems «Aisem 2004», edited by C. Di NAtale, A. D'Amico, G. Martinelli, M.C. CARotta and V. GuIDI (World Scientific Publisher), 329-336
HiLl, K.O. and G. Meltz (1997): Fiber Bragg Grating technology fundamentals and overview, J. Lightwave Technol., 15 (8), 1263

Kersey, A.D., T.A. Berkoff and W.W. Morey (1992): High-resolution fibre-grating based strain sensor with interferometric wavelength-shift detection, Electron. Lett., 28 (3), 236-238.

WENZ, G.M. (1962): Acoustic ambient noise in the ocean: spectra and sources, J. Acoust. Soc. Am., 34, 1936-1956.

Xu, M.G., L. ReEkie, Y.T. Chow and J.P. DAKIN (1993): Optical in-fibre grating high pressure sensor, Electron. Lett., 29, 398-399.

(received September 6, 2005; accepted May 17, 2006) 